\title{
Um Serviço de Representação Ontológica de Contexto Baseada no Padrão de Projeto Estímulo-Sensor-Observação
}

\author{
Ernesto Fonseca Veiga ${ }^{1}$, Renato de Freitas Bulcão Neto ${ }^{1}$ \\ ${ }^{1}$ Instituto de Informática - Universidade Federal de Goiás (UFG) \\ Caixa Postal 131 - CEP 74001-970 - Goiânia - GO - Brazil \\ \{ernestofonseca, renato\}@inf.ufg.br
}

\begin{abstract}
Context-aware computing research highlights ontology-based modeling as a dominant technique for context representation, showing the need for evolving in expressiveness and standardization. This paper proposes Hermes Widget, which is a component that provides the ontological context representation service, with support for the Stimulus-Sensor-Observation sensing pattern. This proposal was validated in a monitoring vital signs scenario with contribution of a nursing team. Results highlight the contributions as expressiveness, standardization and interoperability of generated context.
\end{abstract}

Resumo. Pesquisas em Computação Sensível a Contexto destacam a modelagem baseada em ontologias como técnica dominante para representação de contexto, evidenciando a necessidade de uma evolução quanto a expressividade e padronização. Este trabalho propõe Hermes Widget, um componente que oferece o serviço de representação ontológica de contexto, baseada no padrão de sensoreamento Estímulo-Sensor-Observação. A proposta foi validada em um cenário de monitoramento de sinais vitais, com contribuição de uma equipe de Enfermagem. Os resultados obtidos destacam as contribuições quanto a padronização, expressividade e interoperabilidade do contexto gerado.

\section{Introdução}

O projeto e os serviços realizados por um Sistema de Gerenciamento de Contexto (CMS), de maneira geral, adotam um ciclo de vida ao qual as informações de contexto estão submetidas. Uma abordagem abrangente para o ciclo de vida do contexto, apresentada por [Perera et al. 2014], tem início com a etapa de aquisição, que é seguida pelas etapas de modelagem e de raciocínio, finalizando-se com a disseminação do contexto.

Grande parte da pesquisa que envolve este ciclo de vida está voltada para a investigação de abordagens para a etapa de modelagem [Bettini et al. 2010]. Algumas das principais necessidades identificadas para esta etapa são a evolução quanto à expressividade e à utilização de padrões para a representação de informação contextual [Sehic et al. 2012]. Neste sentido, as tecnologias da Web Semântica, tais como ontologias e a padronização para descrição de informações e realização de consultas, podem contribuir significativamente para a representação e o gerenciamento do contexto.

A importância e os benefícios de uma representação formal das informações de contexto têm sido destacados por [Perera et al. 2014], que recomendam a modelagem baseada em ontologias para apoiar requisitos como expressividade e interoperabilidade. A padronização e o formalismo oferecidos por esta técnica de modelagem permitem 
tanto uma representação quanto o processamento de consultas mais expressivas, além da realização de inferências automáticas de novas informações sobre o contexto.

Neste trabalho apresentamos Hermes Widget, um componente de software que realiza a etapa de modelagem do ciclo de vida do contexto, e implementa como principal serviço a representação de contexto baseada em ontologias. Este componente é fundamentado na utilização de padrões da Web Semântica e também no padrão para modelagem de dados de contexto do domínio de sensoreamento, denominado EstímuloSensor-Observação, com o objetivo de fornecer um modelo de contexto padronizado e um serviço de representação ontológica reutilizável e interoperável para demais componentes de software e aplicações.

Oferecendo um serviço de representação de contexto independente de domínio, Hermes Widget pode ser reutilizado para diferentes cenários de aplicação, sendo ainda parte integrante do projeto do CMS Hermes [Veiga et al. 2014], que contempla todas as etapas do ciclo de vida do contexto. Porém, como possui um projeto arquitetural independente dos demais componentes dessa infraestrutura, o componente pode ser integrado a outros sistemas para realização dos serviços relacionados à etapa de modelagem.

O restante deste trabalho está assim estruturado: a Seção 2 contempla os principais fundamentos relacionados à proposta e introduz o CMS Hermes; a Seção 3 apresenta o componente Hermes Widget, sua arquitetura, requisitos e detalhes de implementação; a Seção 4 discorre sobre os experimentos e a validação do serviço de representação; a Seção 5 apresenta os trabalhos relacionados; e a Seção 6 sintetiza e conclui este trabalho.

\section{Fundamentação Teórica}

Esta seção apresenta a ontologia SSN e o CMS Hermes, introduzindo as informações necessárias para a compreensão deste trabalho.

\subsection{Ontologia Semantic Sensor Network}

Definições semânticas compartilhadas contribuem tanto para a integração do contexto adquirido de diferentes fontes, como para a interoperabilidade deste contexto entre sistemas e aplicações. Neste intuito, a Semantic Sensor Network (SSN) é uma ontologia desenvolvida pelo W3C Incubator Group, que tem como objetivo central descrever um sistema ou processo de sensoreamento, bem como os demais conceitos e relações deste domínio, conforme o padrão de projeto ontológico Estímulo-Sensor-Observação, ou Stimulus-Sensor-Observation (SSO) [Janowicz and Compton 2010].

O padrão de projeto SSO tem como objetivo apoiar a representação semântica de sistemas e dados de sensoreamento, e foi criado com base em princípios ontológicos que o tornam reutilizável para diferentes domínios de aplicação [Janowicz and Compton 2010]. Independente de ontologias de alto nível, introduz um conjunto de classes e relações, sendo as suas principais definições:

- Estímulos: mudanças detectáveis no ambiente, seja este físico ou lógico. São o ponto de partida de uma medição, podendo ser direta ou indiretamente relacionadas a propriedades ou características de interesse. Exemplos de estímulos podem ser alterações nos sinais vitais de um paciente.

- Sensores: podem ser componentes físicos ou de software que realizam o sensoreamento de um estímulo, por exemplo, um sensor de temperatura corpórea. 
- Observações: atuam como a relação entre um estímulo de entrada, o sensor que o coleta e a saída deste sensor. Podem também descrever outros parâmetros, tais como espaço (localização) e tempo (instante de uma aferição). Uma observação é o conjunto das informações que descrevem o contexto do sensoreamento.

A Figura 1 apresenta a implementação do padrão SSO na ontologia SSN, com seus principais conceitos e relações.

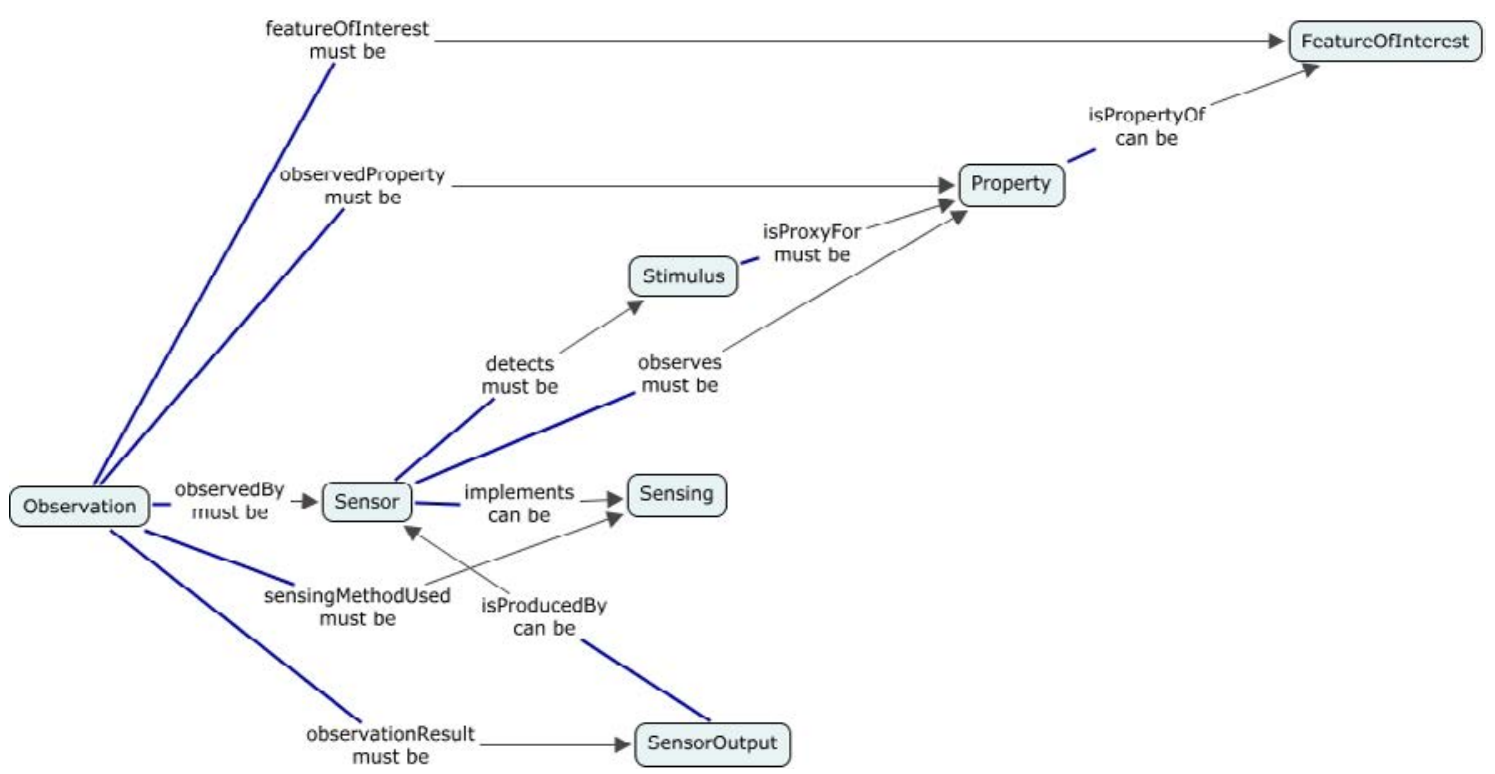

Figura 1. Padrão Stimulus-Sensor-Observation [Janowicz and Compton 2010]

A ontologia SSN foi utilizada como base para a padronização do serviço de representação ontológica de contexto, núcleo da proposta deste trabalho.

\subsection{Sistema para Gerenciamento de Contexto Hermes}

Hermes é um CMS baseado em ontologias proposto por [Veiga et al. 2014], que tem como objetivo principal apoiar o desenvolvimento de aplicações sensíveis a contexto. Os principais requisitos para sua construção são: $i$ ) oferecer serviços de apoio ao gerenciamento do ciclo de vida das informações de contexto; ii) infraestrutura baseada em componentes independentes; e iii) serviços apoiados na semântica das informações de contexto; destacados por [Bettini et al. 2010, Perera et al. 2014].

A Figura 2(a) apresenta a arquitetura de Hermes e seus componentes: i) Hermes Base, responsável pela comunicação componente/componente e componentes/aplicações na disseminação de contexto [Maranhão et al. 2014]; ii) Hermes Widget, representação ontológica de contexto coletado de sensores [Veiga et al. 2016]; iii) Hermes Aggregator, agregador para geração de contexto com maior nível de expressividade; iv) Hermes Interpreter, interpretação e filtragem semântica de contexto [Maranhão et al. 2014]; e v) Hermes History, gerenciador de histórico, consultas e acesso a informação de contexto.

No intuito de simplificar a visualização do fluxo de contexto dentro do CMS Hermes, a Figura 2(b) apresenta separadamente o esquema de comunicação. O componente Hermes Base, responsável pela comunicação em Hermes, encapsula o acesso a 


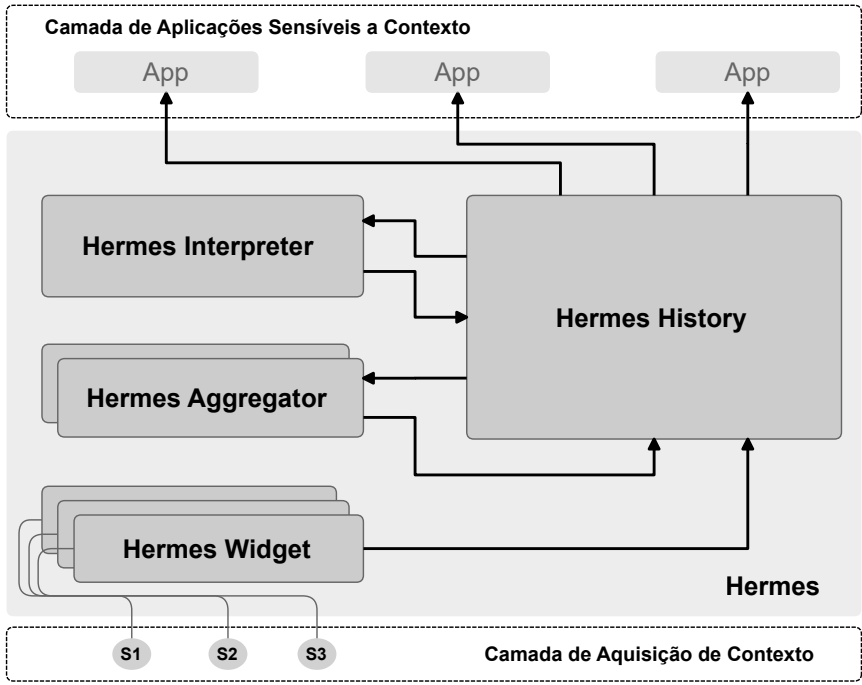

(a)

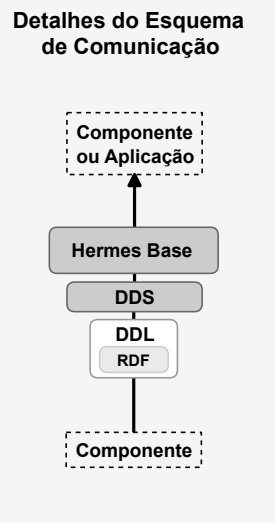

(b)

Figura 2. Infraestrutura Hermes, adaptada de [Veiga et al. 2016], onde: (a) apresenta o projeto arquitetural de componentes e (b) o esquema de comunicação.

um middleware baseado na especificação Data Distribution Service (DDS) ${ }^{1}$, que permite a comunicação publish-subscribe do contexto serializado no formato RDF, padrão para representação de informações na Web Semântica. Este esquema é utilizado na arquitetura de Hermes para comunicação entre os componentes e também destes com as aplicações.

\section{Hermes Widget: Representação Ontológica e Padronização de Contexto}

Hermes Widget é o componente responsável por abstrair o serviço de representação ontológica do contexto. Este serviço é fundamental dentro do CMS Hermes (ou de qualquer outra infraestrutura de contexto), uma vez que oferece as informações de entrada necessárias à realização dos demais serviços e também às aplicações. A representação ontológica marca em Hermes a primeira etapa de transformação dos dados sem processamento, adquiridos de sensores, em contexto de alto nível, que pode ser utilizado por outros componentes e por aplicações sensíveis a contexto.

\subsection{Requisitos e Arquitetura}

Os principais requisitos de Hermes Widget são:

1. Permitir a especificação de um sensor e do processo de sensoreamento: o componente de deve permitir a descrição de um processo de sensoreamento e de suas relações, tratando termos e eventos deste domínio tais como: sensor, propriedade, entidade de interesse, observação (ou sensoreamento), resultado entre outros.

2. Representação ontológica das informações de contexto: a representação realizada por Hermes Widget deve representar formalmente as informações de contexto sendo, neste caso, baseada em ontologias.

3. Padronização do modelo de contexto: Hermes Widget deve permitir a representação padronizada do contexto, independente do domínio de aplicação.

\footnotetext{
${ }^{1}$ DDS: http: //www.omg.org/spec/DDS/
} 
4. Disseminação de informações de contexto: após representar o contexto recebido da camada de aquisição, o Hermes Widget deve disponibilizar essas informações para demais componentes do CMS Hermes e/ou aplicações interessadas.

A ontologia SSN e o padrão SSO foram definidos para cumprir os requisitos de 1 a 3, oferecendo a base para a construção do modelo de representação de contexto de Hermes Widget e também um padrão para esta representação (SSO). A representação baseada em ontologias, cumprindo o requisito 2, atribui formalidade e expressividade ao contexto, permitindo a descrição semântica das informações e a realização de inferências. Este mesmo requisito corrobora com o requisito 3, uma vez que, tanto a representação baseada em ontologias quanto a padronização de um modelo para a descrição do contexto, garantem a interoperabilidade no processo de disseminação.

A Figura 3 apresenta as camadas arquiteturais do componente Hermes Widget. Este projeto foi definido com base nos requisitos apresentados, juntamente com os requisitos do próprio CMS Hermes, que definem os serviços de configuração, comunicação e notificação de maneira geral para todos os seus componentes.

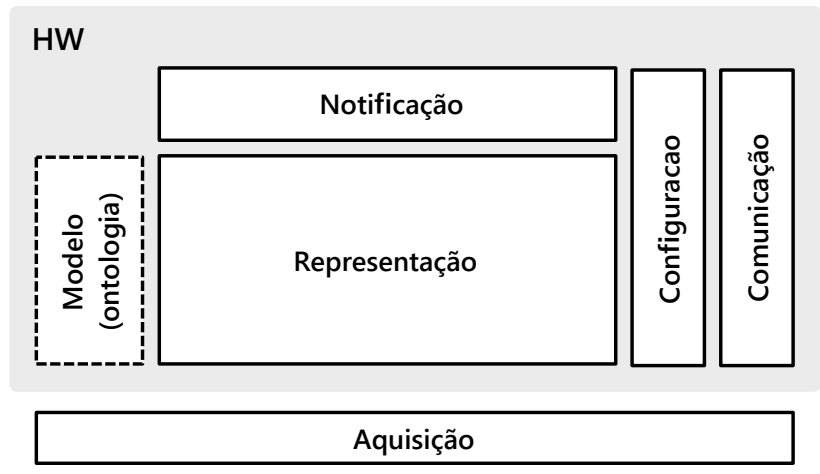

Figura 3. Arquitetura em camadas do componente Hermes Widget

A camada de aquisição, externa ao Hermes Widget, recebe as alterações de contexto detectadas pelos sensores, transmitindo-as à camada de representação ${ }^{2}$. A representação é realizada com base em um modelo ontológico, conforme especificado nos requisitos, e o contexto produzido é gerenciado pelas camadas de configuração, comunicação e notificação, que realizam os serviços necessários à disponibilização do contexto representado.

A camada de configuração de um Hermes Widget acessa um arquivo de definição $J S O N$, que descreve os parâmetros de configuração do componente. Estas definições tratam de maneira específica da criação do tópico que receberá as informações de contexto representadas e que será assinado por aplicações e pelos componentes interessados, que passarão a receber as notificações de contexto desse tópico.

\subsection{Serviço de Representação Ontológica}

A Figura 4 apresenta os detalhes relacionados ao projeto e implementação do serviço de representação ontológica de contexto. Cada aferição de contexto recebida de um sensor, através da camada de aquisição, é transformada em uma informação de entrada para o serviço de representação, que é composto de duas partes principais:

\footnotetext{
${ }^{2}$ Hermes Widget e nenhum outro componente de Hermes trata a aquisição de dados de sensores físicos.
} 
1. Gerente de Representação: é a subcamada em que se realiza a representação ontológica do contexto adquirido. Tem como base a ontologia SSN, que fornece o modelo de contexto para as informações adquiridas.

2. Gerente de Contexto: prepara as representações para envio, na forma de instâncias de contexto devidamente serializadas.

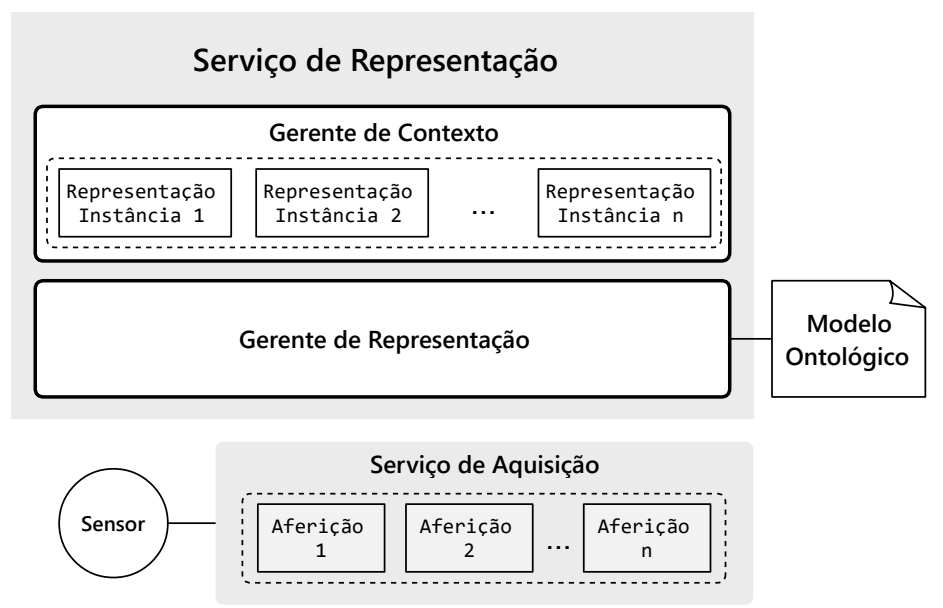

Figura 4. Serviço de representação ontológica de contexto

Na implementação do serviço de representação de contexto foi utilizado o framework Jena ${ }^{3}$, da Apache, tecnologia open source que oferece API's para a manipulação de informação semântica, tais como modelos RDF, ontologias OWL e realização de consultas SPARQL. A ontologia SSN é o modelo utilizado para criação das representações de contexto, tornando o serviço reutilizável para receber entradas de diferentes tipos de sensores e produzir como saída uma representação de contexto padronizada.

\section{Cenário de Validação do Hermes Widget}

Essa seção apresenta a validação de Hermes Widget por meio da simulação de um cenário de monitoramento de sinais vitais humanos em Unidade de Terapia Intensiva (UTI).

\subsection{Cenário de testes}

Para esta validação foram implementados cinco Hermes Widgets, sendo um para cada sinal vital, a saber: pressão arterial, temperatura corpórea, frequência de pulso, frequência respiratória e saturação de oxigênio. O objetivo foi reproduzir o ambiente de monitoramento real de uma UTI.

Não dispondo de sensores físicos para realização dos testes, nem do acesso à uma UTI real para integração com as instâncias do componente Hermes Widget, o serviço de aquisição foi realizado na forma de simulação. Essa simulação ocorreu através da leitura dos dados da base pública PhysioBank ${ }^{4}$, que disponibiliza monitoramento de sinais vitais reais coletados de pacientes de UTI. Dessa forma, foram implementados sensores virtuais que importam os dados disponíveis na base de acordo com o seu formato nativo e

\footnotetext{
${ }^{3}$ Jena: https://jena.apache.org/

${ }^{4}$ PhysioBank: https://physionet.org/mimic2/
} 
disponibilizam as aferições de acordo com a frequência de leitura especificada, simulando o comportamento de um sensor físico.

A Tabela 1 contém amostras de exemplo dos registros de um paciente identificado por $033 n$, coletados da PhysioBank. Essa amostra possui frequência de monitoramento definida com intervalo de 20 segundos entre aferições, considerada de alta precisão pela Equipe de Enfermagem do Hospital das Clínicas (UFG), que apoiou este projeto. A primeira coluna apresenta o momento da medição a partir do início do monitoramento, e as demais apresentam os dados aferidos para cada sinal vital, representado pelas suas respectivas siglas:

-ABPdias: Pressão sanguínea diastólica -ABPsys: Pressão sanguínea sistólica

- ABPMean: Pressão sanguínea média - Temp: Temperatura corpórea

- Pulse: Frequência de pulso

- Resp: Frequência respiratória

- SpO2: Saturação de oxigênio

Tabela 1. Amostra de registro da base MIMIC para o paciente 033n.

\begin{tabular}{|c|c|c|c|c|c|c|c|}
\hline Intervalo & ABPMean & ABPsys & ABPdias & Pulse & Resp & SpO2 & Temp \\
\hline sec & $\mathrm{mmHg}$ & $\mathrm{mmHg}$ & $\mathrm{mmHg}$ & $\mathrm{bpm}$ & $\mathrm{bpm}$ & $\%$ & ${ }^{\circ} \mathrm{C}$ \\
\hline 0 & 81 & 138 & 54 & 53 & 25 & 94 & 37.5 \\
\hline 20 & 78 & 134 & 53 & 53 & 30 & 94 & 37.5 \\
\hline 40 & 79 & 135 & 53 & 52 & 36 & 94 & 37.5 \\
\hline 60 & 78 & 133 & 53 & 52 & 26 & 94 & 37.5 \\
\hline
\end{tabular}

\subsection{Validação Funcional da Representação Ontológica}

Como descrito anteriormente, o serviço de representação realizado pelo componente Hermes Widget utiliza a padronização Estímulo-Sensor-Observação (Figura 1), adotada pela ontologia SSN, no intuito de representar de maneira expressiva e interoperável as informações de um sensoreamento. Para cada informação de contexto aferida é criado um indivíduo do tipo ssn:Observation, ao qual serão adicionadas as propriedades:

- ssn:observes: que relaciona um sensor ssn:SensingDevice a uma propriedade observada ssn:Property;

- ssn:isProducedBy: relaciona a informação produzida ssn:SensorOutput ao sensor ssn:SensingDevice que a produz;

- ssn:observedBy e ssn:observationResult: ligam respectivamente um sensor e a informação produzida por ele, a uma observação ssn:Observation.

A Figura 5 apresenta o grafo gerado a partir do modelo de contexto armazenado no repositório que contém a persistência do sensoreamento de duas instâncias de medição de pressão sanguínea ${ }^{5}$.

Neste exemplo a propriedade do tipo ssn:Property descrita é a pressão sanguínea, representada pela instância ssn:BloodPressure. Os sensores virtuais que observam esta propriedade são do tipo ssn:SensingDevice, e são descritos pelas instâncias ssn:BloodPressureSensor001 e ssn:BloodPressureSensor002. O fato de existirem dois

\footnotetext{
${ }^{5}$ Por questões de espaço o modelo RDF que deu origem ao grafo não será apresentado. Este grafo representa o modelo na sua integridade.
} 


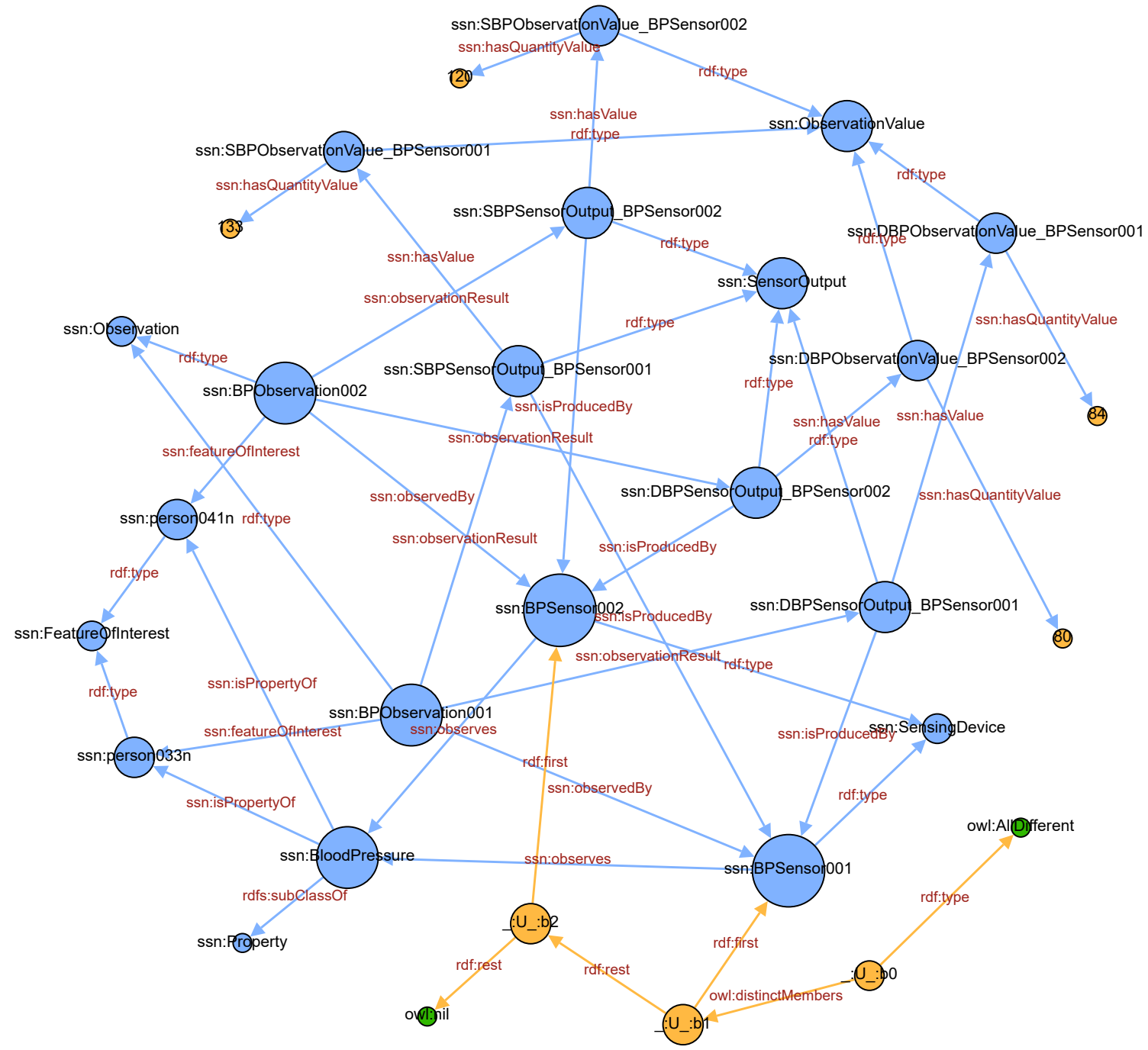

Figura 5. Grafo da representação SSN para duas instâncias de pressão arterial

sensores distintos para o mesmo tipo de sinal vital nesta representação significa que estas foram coletadas de pacientes diferentes.

Os resultados do monitoramento de contexto representado são apresentados por indivíduos do tipo ssn:SensorOutput, que são as saídas resultantes das aferições, que incluem os valores observados ssn:has Value, que são representados pelos sensores e possuem um valor observado ssn:ObservationValue para cada medição aferida, neste caso $(133,84)$ e $(120,80)$. Todas estas propriedades são relacionadas em uma instância da classe ssn:Observation, que descreve o monitoramento realizado.

O padrão de projeto Estímulo-Sensor-Observação, implementado pela ontologia SSN, contribui em Hermes Widget para a definição de uma representação de contexto padronizada, que pode ser utilizada independente do domínio de aplicação. Dessa forma, além de oferecer grande facilidade para o compartilhamento das informações representadas, qualquer componente ou aplicação que utilize o contexto gerado por Hermes Widget tratará a informação de maneira análoga, seguindo o padrão que rege a sua estrutura. Portanto, além do formato de representação do contexto, a estrutura semântica também é 
padronizada, independente da informação de contexto a ser representada.

Também foram realizados outros experimentos, como uma avaliação da escalabilidade do serviço de representação ontológica de contexto. Como resultado, verificou-se a capacidade do Hermes Widget em processar grandes volumes de dados sem impactos no tempo de processamento por representação, como descrito em [Veiga et al. 2016].

\section{Trabalhos Relacionados}

LoCCAM (Loosely Coupled Context-Aware Middleware) [Maia et al. 2013] é um middleware de arquitetura orientada a componentes que propõe a redução do acoplamento entre o processo de aquisição de contexto e as aplicações que o utilizam. Os componentes Context Acquisition Components (CACs) encapsulam o código de acesso a sensores para fornecer informação de contexto de alto nível.

Segundo o princípio de reusabilidade, CroCo (Cross-application Context management Service) [Mitschick et al. 2010] destaca a relevância de um sistema de representação independente de domínio, apresentando um modelo ontológico de propósito geral que trata os principais aspectos do contexto. Porém, CroCo assume que seus provedores já enviem o contexto na forma de triplas RDF, que é recebido pelo serviço Context Update Service. CroCo não define um padrão para a representação do contexto.

Em infinitum [Jaroucheh et al. 2012], os componentes provedores de contexto (CP's) realizam a representação de informações recebidas, gerando unidades de contexto chamadas Contextlets, que são uma parte específica da informação sobre uma determinada entidade. Uma limitação de infinitum é a falta de um processo para tornar a representação de contexto flexível em sua arquitetura.

O componente Hermes Widget possui uma ideia similar à dos CAC's de LoCCAM e da estrutura de CroCO e de infinitum quanto à separação entre aquisição/representação e utilização do contexto. Porém, diferente de LoCCAM, esta proposta utiliza a modelagem baseada em ontologias para oferecer maior expressividade e independência do modelo de contexto em relação a representação baseada em espaço de tuplas, realizada pelos CAC's. Diferendo em relação aos demais trabalhos relacionados, nesta proposta é apresentada a padronização da estrutura de representação ontológica de contexto, neste caso utilizando o padrão para sensoreamento Estímulo-Sensor-Observação.

\section{Conclusões}

Este artigo apresentou o componente Hermes Widget, que recebe dados de sensores e, através do processo de representação ontológica, gera um modelo de contexto altamente expressivo e em um formato de representação com apoio à interoperabilidade.

Como contribuições deste trabalho, destacam-se: $i$ ): um serviço de representação ontológica de contexto reutilizável e independente de domínio de aplicação; ii) modelo de representação de contexto padronizado de acordo com a ontologia SSN (padrão SSO); iii) representação de contexto compartilhável e interoperável entre sistemas e aplicações; e $i v$ ) validação da representação de contexto no cenário de monitoramento de sinais vitais humanos, apoiada por uma base de dados com aferições reais de sinais vitais de pacientes e uma equipe de enfermagem com experiência em UTI. 
Destaca-se como trabalho futuro a utilização do componente Hermes Widget e do serviço de representação em outros cenários de aplicação além do monitoramento de sinais vitais. Também devem ser implementadas outras aplicações sensíveis a contexto que utilizam as informações representadas em diferentes domínios, no intuito de avaliar extensivamente a reusabilidade e a flexibilidade do componente proposto.

\section{Agradecimentos}

O autor Ernesto Fonseca Veiga agradece a CAPES pela bolsa de mestrado concedida a esta pesquisa.

\section{Referências}

Bettini, C., Brdiczka, O., Henricksen, K., Indulska, J., Nicklas, D., Ranganathan, A., and Riboni, D. (2010). A survey of context modelling and reasoning techniques. Pervasive Mob. Comput., 6(2):161-180.

Janowicz, K. and Compton, M. (2010). The stimulus-sensor-observation ontology design pattern and its integration into the semantic sensor network ontology. In 3rd International Conference on Semantic Sensor Networks - Volume 668, pages 64-78.

Jaroucheh, Z., Liu, X., and Smith, S. (2012). An approach to domain-based scalable context management architecture in pervasive environments. Personal Ubiquitous Comput., 16(6):741-755.

Maia, M. E. F., Fonteles, A., Neto, B., Gadelha, R., Viana, W., and Andrade, R. M. C. (2013). Loccam - loosely coupled context acquisition middleware. In 28th Annual ACM Symposium on Applied Computing, pages 534-541.

Maranhão, G. M., Sene Júnior, I. G., and Bulcão Neto, R. F. (2014). Anatomy of a semantic context interpreter with real-time events notification support. In 20th Brazilian Symposium on Multimedia and the Web, pages 159-162.

Mitschick, A., Pietschmann, S., and Mei $\beta$ ner, K. (2010). An Ontology-Based, CrossApplication Context Modeling and Management Service. Int. J. Semant. Web Inf. Syst., $6(1): 39-54$.

Perera, C., Zaslavsky, A., Christen, P., and Georgakopoulos, D. (2014). Context aware computing for the internet of things: A survey. Communications Surveys Tutorials, IEEE, 16(1):414-454.

Sehic, S., Li, F., Nastic, S., and Dustdar, S. (2012). A programming model for contextaware applications in large-scale pervasive systems. In IEEE 8th International Conference on Wireless and Mobile Computing, Networking and Communications, pages 142-149.

Veiga, E. F., Maranhão, G. M., and Bulcão Neto, R. F. (2016). Development and scalability evaluation of an ontology-based context representation service. IEEE Latin America Transactions, 14(3):1380-1387.

Veiga, E. F., Maranhão, G. M., and Bulcão Neto, R. F. (2014). Apoio ao desenvolvimento de aplicações de tempo real sensíveis a contexto semântico. In IX Workshop de Teses e Dissertações do XX Simpósio Brasileiro de Sistemas Multimídia e Web, pages 1-4. 